\title{
Análisis farmacobotánico del órgano subterráneo del "coro" (Nicotiana paa, Solanaceae), un fumatorio sagrado moqoit
}

\author{
Pharmaco-botanical analysis of the subterranean organ of the \\ "coro" (Nicotiana paa, Solanaceae) for moqoit sacred smoking
}

D.o.I.: doi.org/10.30550/j.lil/2018.55.2/1

\author{
Anconatani, Leonardo M. ${ }^{1 *}$; Rafael A. Ricco ${ }^{1}$; Gustavo F. Scarpa ${ }^{2}$; \\ Marcelo L. Wagner ${ }^{1}$ \\ 1 Universidad de Buenos Aires. Facultad de Farmacia y Bioquímica. Departamento de Farmacología. \\ Cátedra y Museo de Farmacobotánica "Juan A. Domínguez". Junin 956, (1113) Ciudad Autónoma de \\ Buenos Aires. República Argentina. \\ 2 Museo Argentino, de Ciencias Naturales "Bernardino Rivadavia". Av. Ángel Gallardo 470, (1405) Ciudad \\ Autónoma de Buenos Aires. República Argentina. \\ * Autor corresponsal: Imanconatani@ffyb.uba.ar
}

\begin{abstract}
Resumen - "Coro" (Nicotiana paa Mart. Crov.) es una planta que ha sido documentada, a lo largo del tiempo por distintas fuentes históricas, etnográficas y etnobotánicas. Su órgano subterráneo es y ha sido empleado como fumatorio y medicina por parte de los moqoit y qom del Chaco Argentino, como así también por otros pueblos originarios de la misma región. El objetivo de este trabajo se centró en realizar la caracterización morfo-anatómica del órgano subterráneo de esta planta, empleando distintas técnicas histológicas e histoquímicas para tal fin. Los resultados obtenidos nos permiten afirmar que estamos en presencia de un tallo secundario, subterráneo, cuya función principal es la reserva. Por otro lado, la caracterización del almidón de esta especie resulta de gran utilidad para poder identificar la presencia del rizoma de "coro", no solo en objetos de la cultura material moqoit, sino también, en restos arqueológicos de diversos grupos humanos en los cuales este vegetal ha sido empleado. Este estudio cobra importancia debido a que no se halla hasta la fecha ningún análisis de este tipo.

Palabras clave: Rizoma, Almidón, mocovíes.
\end{abstract}

Abstract - "Coro" (Nicotiana paa Mart. Crov.) is a plant that has been documented over time by different historical, ethnographic and ethnobotanical sources. Its underground organ is and has been used for smoking and medicine by the Moqoit and Qom of the Argentine Chaco, as well as by other indigenous peoples of the same region. The aim of this work was to perform a morpho-anatomical characterization of the subterranean organ of this plant, using different histological and histochemical techniques for this purpose. The results obtained allow us to affirm that this organ is a secondary stem, subterranean, whose main function is reservation. On the other hand, the characterization of the starch in this species is very useful to identify the presence of the "Coro" rhizome, not only in objects of the Moqoit

\footnotetext{
> Ref. bibliográfica: Anconatani, L. M.; Ricco, R. A.; Scarpa, G. F.; Wagner, M. L. 2018. Análisis farmacobotánico del órgano subterráneo del "coro" (Nicotiana paa, Solanaceae), un fumatorio sagrado moqoit. Lilloa 55 (2): 1-7.

> Recibido: 18/09/18 - Aceptado: 09/11/18

$>$ URL de la revista: http://lilloa.lillo.org.ar

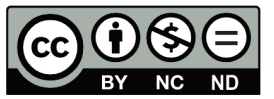


material culture, but also in archaeological remains of different human groups in which this vegetable has been used. This study is important because no analysis of this type has been found to date.

Keywords: Rhizome, Starch, Mocovies.

\section{INTRODUCCION}

El «coro» (Nicotiana paa Mart. Crov.) (Solanaceae), es una planta que ha sido documentada desde antaño por los misioneros jesuitas (Montenegro, 1945; Jolis, 1972; Dobrizhoffer, 1967, Lozano, 1875; Ruiz Moreno, 1948; Deckmann Fleck, 2015), como así también por antropólogos (Serrano, 1934; Métraux, 1946; Pérez Gollán y Gordillo, 1993), botánicos (Schulz, 1976; Cárdenas, 1968; Zardini, 1976-1977; entre otros) y con mayor profundidad, por etnobotánicos como Martinez Crovetto (1978; 1980) y Scarpa y Rosso (2011), debido a su empleo de carácter mágico, religioso y medicinal por distintos grupos chaqueños como los qom, abipón, wichí, lule y moqoit principalmente y, además, otros pueblos como los andinos,los guaraníes y los mapuche (Martínez Crovetto, 1980).

Los estudios antes mencionados son los únicos que registran, el empleo de este vegetal, principalmente por parte de los originarios moqoit del Chaco Argentino.

Según Martínez Crovetto (1980) los moqoit emplean los rizomas secos para fumar en pipa. Por otro lado, Scarpa y Rosso (2011) indican que los indígenas moqoit fuman el órgano subterráneo del «coro» — previamente secado- tanto en contextos ceremoniales como extra-ceremoniales. El primero de los casos incluye tanto reuniones o «juntas» $\mathrm{y}$ celebraciones colectivas, como los rituales chamánicos. Durante las celebraciones colectivas los «pioGonak» o chamanes moqoit advierten que mediante su empleo adquieren «más poder para curar» ya que les facilita la invocación a espíritus auxiliares necesarios para ello. En ámbitos extra-ceremoniales, en cambio, suelen emplearlo hombres adultos mayores, quienes reconocen que tiene la «capacidad de otorgar fortaleza a quien lo fuma» (Scarpa y Rosso, 2011). Estos autores y Martínez Crovetto (1980) indican que esta planta ha sido empleada frecuentemente mezclada con las hojas de tabaco (Nicotiana sp.), o conjuntamente a otras plantas, a modo de atemperantes o aromatizantes.

La identidad botánica del «coro», sin embargo, fue una incógnita durante mucho tiempo, hasta que Martínez Crovetto la describiera e identificara como una nueva especie (Martinez Crovetto, 1978). Posteriormente, sus datos fueron confirmados y ampliados gracias a nuevos trabajos de campo en los que se documentó su identidad, se describieron aspectos novedosos sobre la autoecología de esta especie, así como de su obtención, procesamiento y consumo entre los indígenas moqoit (Scarpa y Rosso, 2011).

Este trabajo tiene como objetivo realizar un análisis morfoanatómico y micrográfico del órgano subterráneo del «coro» (Nicotiana paa Mart. Crov.) (Solanaceae) debido a que no se ha efectuado hasta la fecha ningún análisis de este tipo.

\section{MATERIALES Y MÉTODOS}

\section{Materiales}

Se emplearon dos muestras correspondientes a los órganos subterráneos del «coro» (Nicotiana paa Mart. Crov.) (Solanaceae), colectados el XI-2008, Colonia Necochea, Dpto. Chacabuco, y el 14-XII-2010, en el Departamento de General Pinedo, Provincia de Chaco, Argentina.

Los ejemplares de herbario corresponden a: ARGENTINA. Prov. Chaco, Dpto. General Pinedo, 14-XII-2010, Scarpa 798 (BA) y ARGENTINA. Prov. Chaco, Dpto. Chacabuco, Colonia Necochea, XI-2008, Rosso s/n (BA).

\section{MÉTOdos}

Se utilizaron las dos muestras con el objeto de establecer los caracteres morfoanató- 
micos correspondientes al órgano subterráneo. Se realizaron los siguientes estudios:

\subsection{Análisis macroscópico.— Se procedió} a la observación macroscópica con el objeto de detallar sus caracteres morfológicos externos.

2.2. Cortes y coloración.- Los cortes se efectuaron en la región media del órgano subterráneo, seguido del vaciado y la doble coloración diferencial. Previamente a ello, se le realizó un ablandamiento a un fragmento del material sometiéndolo a ebullición en agua durante 5 min. Posteriormente se realizaron varios cortes transversales con el empleo de un micrótomo de deslizamiento marca Reichert, a un espesor de $50 \mu \mathrm{m}$. Estos cortes fueron posteriormente sumergidos en una solución al $50 \%$ de hipoclorito de sodio para vaciarlos de su contenido celular, hasta observar transparencia. Luego se sometió a dichos cortes a una deshidratación mediante pasajes sucesivos a través de soluciones de etanol de concentración creciente y la posterior coloración con safranina y fast green (Zarlavsky, 2014). Por último, los transcortes se montaron en un portaobjetos y se fijaron con bálsamo de Canadá. Se procedió a su descripción mediante su observación con el microscopio óptico.

La técnica descripta se empleó para cada material analizado.

2.3. Disociación fuerte.- Se empleó el método de Boodle (1916). Se tomó una pequeña cantidad de leño del material vegetal y se introdujo en un recipiente adecuado. Se agregó solución de $\mathrm{KOH}$ al 10\% hasta cubrir el material y se llevó a ebullición durante 10 minutos. Se dejó reposar hasta que disminuyó su temperatura y luego se filtró y se descartó el líquido sobrenadante. Posteriormente se lavó con agua destilada repetidas veces. Se colocó el material retenido en un vaso de precipitados y se cubrió con una solución de ácido crómico al 25\% y se dejó actuar a temperatura ambiente durante $60 \mathrm{~min}$. Una vez ablandado el material, se procedió a lavarlo reiteradas veces con agua destilada. Se montó el disociado obtenido entre portaobjetos y cubreobjetos y se observó con el microscopio. (D'Ambrogio de Argüeso, 1986)

La técnica descripta se empleó para cada material analizado.

2.4. Disociación leve.- Se tomó una pequeña cantidad de corteza del material vegetal y se agregó solución de $\mathrm{NaOH}$ al $5 \%$ hasta cubrir el material y se llevó a ebullición durante 5 minutos. Se filtró y se lavó con agua destilada repetidas veces. Posteriormente se montó el disociado obtenido entre portaobjetos y cubreobjetos y se observó con el microscopio (D'Ambrogio de Argüeso, 1986).

La técnica descripta se empleó para cada material analizado.

2.5. Muestra en polvo.- Se procedió a la obtención de material pulverizado proveniente de la corteza del material vegetal mediante raspado. Se montó la muestra obtenida entre portaobjetos y cubreobjetos en medio acuoso, según Farmacopea Argentina, séptima edición. Se observó con un microscopio de luz blanca, luz polarizada y contraste de fase (DIC). Se realizaron las reacciones histoquímica necesarias para evidenciar la presencia de oxalato de calcio (mediante el empleo de ácido clorhídrico) y de Lugol para almidón (D’Ambrogio de Argüeso, 1986).

Para la descripción de los granos de almidón se siguieron los criterios de «The International Code for Starch Nomenclature» (ICSN, 2011).

Para el análisis microscópico, se empleó un microscopio Carl Zeiss Axioskop 2 Plus provisto de una cámara digital Moticam X2. Se emplearon aumentos totales de 100x, 200x y 400x.

La técnica descripta se empleó para cada material analizado.

\section{RESULTADOS}

\section{CARACTERES MORFOLÓGICOS}

Se trata de un órgano de tipo subterráneo, de entre 100 a $200 \mathrm{~mm}$ de longitud, 


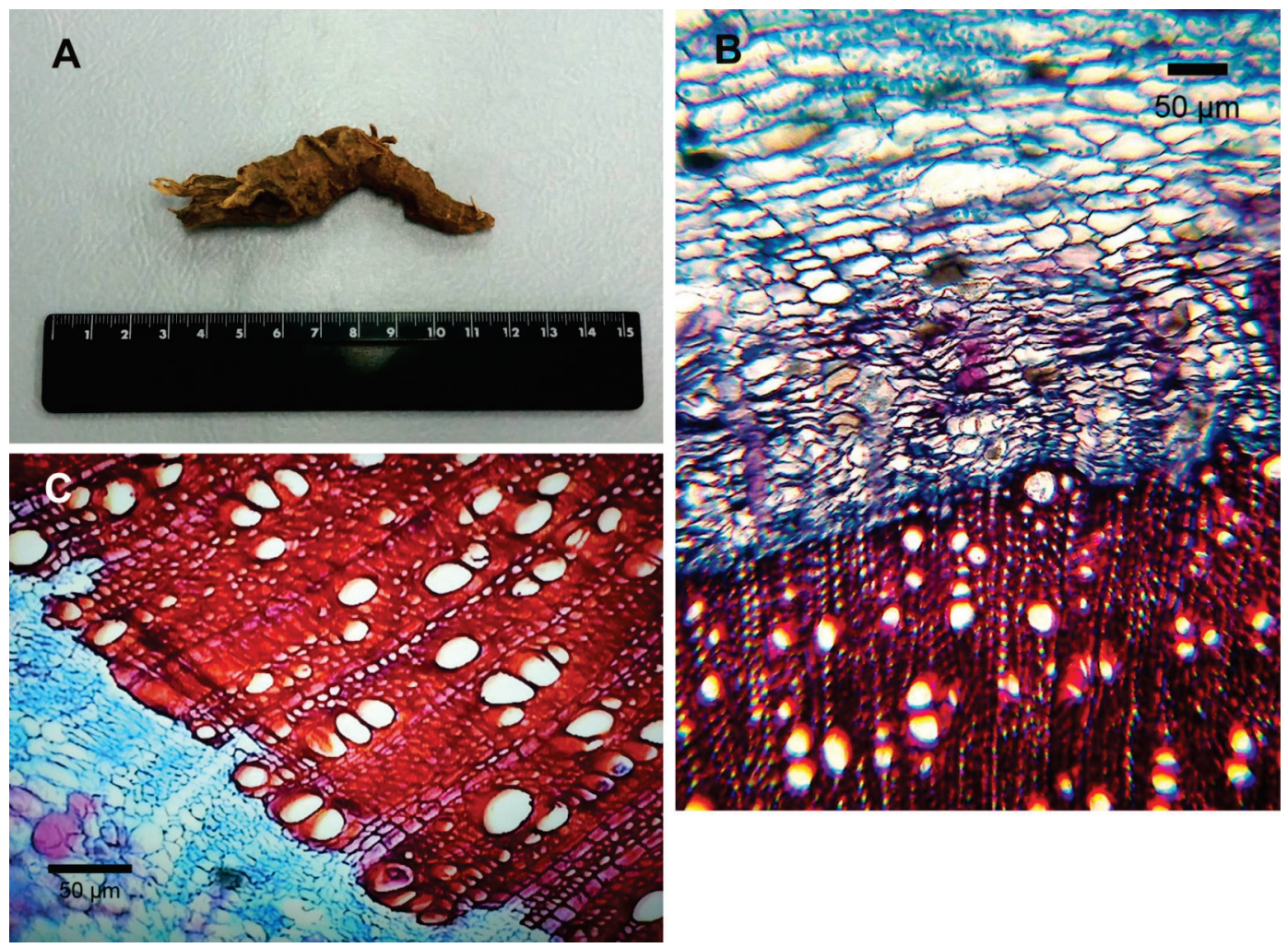

Fig. 1. Nicotiana paa. A] Muestra de Rizoma de Coro. B] Transcorte. C] Tejidos de conducción. Scarpa 798 (BA).

algo carnoso en estado fresco, con corteza de color pardo-rojizo, fuerte e irregularmente estriado cuando seco (Fig. 1A).

\section{TRANSCORTE}

Su sección transversal evidencia una peridermis compuesta principalmente por 15 capas de células suberosas. Inmediatamente después, un parénquima de tipo reservante conformado por 15 a 20 estratos de células isodiamétricas, en donde se evidencia una gran cantidad de granos de almidón de reserva, de dos formas diferentes (descriptos en el polvo de la corteza). En dicho tejido se observan además idioblastos cristalíferos que contienen arenillas cristalinas (compuestas por microcristales de oxalato de calcio). El tejido de conducción se dispone como un sistema vascular continuo con presencia de cambium entre floema y xilema (Fig. 1B, 1C). No se observa la presencia de médula.

\section{DISOCIADO FUERTE DEL LEÑO}

Se observaron abundantes fibras, de 400 $\mu \mathrm{m}$ de longitud promedio, con extremos aguzados, fibro-traqueidas de $300 \mu \mathrm{m}$ de longitud promedio, con puntuaciones simples y unidades de vaso de tipo reticulado de entre $75 \mu \mathrm{m}$ a $100 \mu \mathrm{m}$ de largo por $50 \mu \mathrm{m}$ de diámetro (Fig. 2A) y células parenquimáticas del xilema, de forma rectangular, de $35 \mu \mathrm{m}$ por $100 \mu \mathrm{m}$, con puntuaciones simples.

\section{DISOCIADO LEVE DE LA CORTEZA}

Se observó abundante arenilla cristalina (cristales de oxalato de calcio) (Fig. 2B). Se detectó también la presencia de células suberosas constituido por aproximadamente 15 estratos de células (Fig. 2C) y de dos tipos de esclereidas, una de ellas prismáticas rectangulares de 25 a $35 \mu \mathrm{m}$ de largo (Fig. 2D) y fibroesclereidas de 100 a $120 \mu \mathrm{m}$ de longitud (Fig. 2E). 

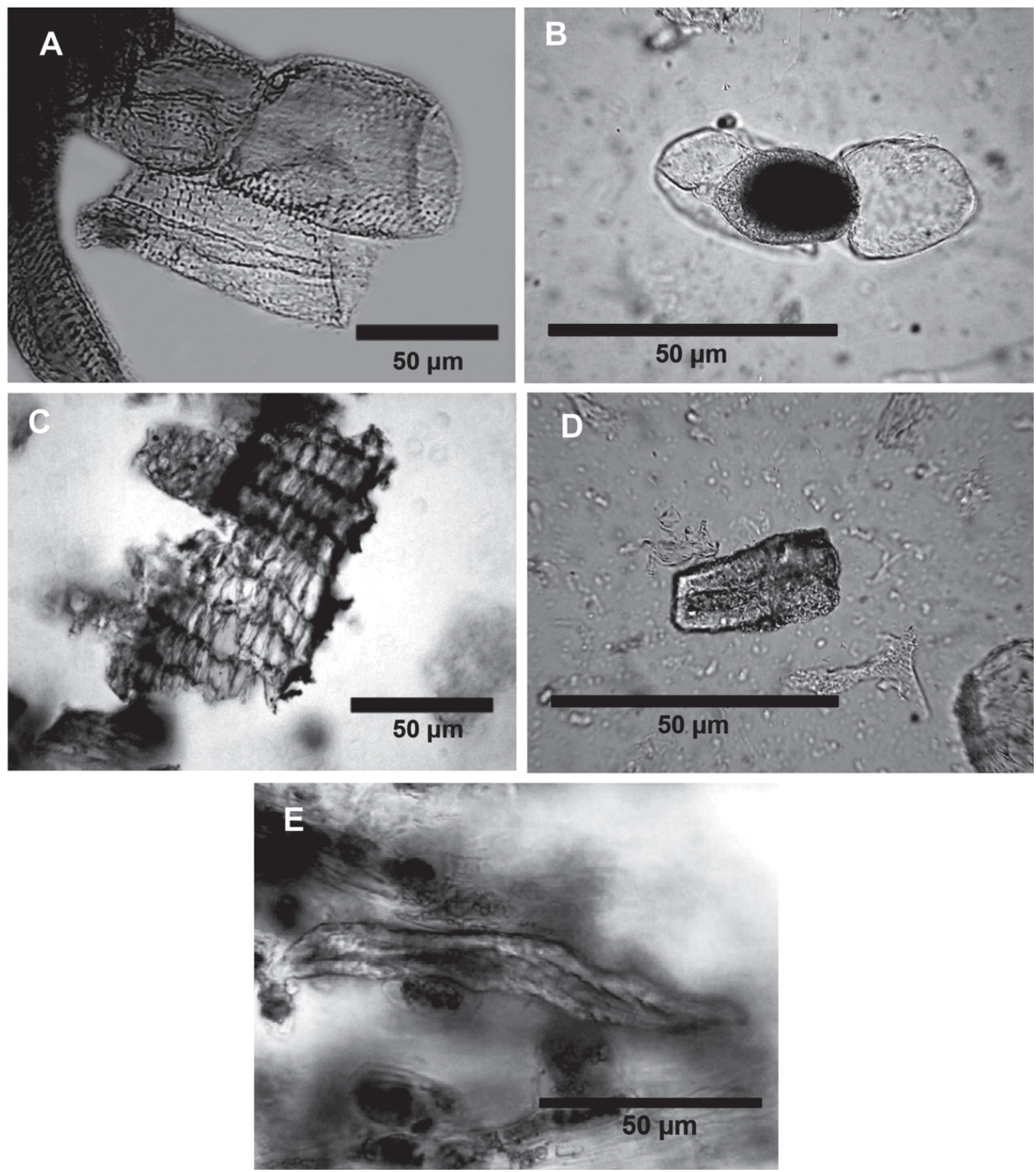

Fig. 2. Nicotiana paa. A) Unidades de Vaso. B] Arenillas cristalinas. C) Súber. D) Escleréida rectangular. E) Fibroescleréida. Scarpa 798 (BA).

\section{POLVO DE LA CORTEZA}

Se observaron dos tipos (bimodal) de granos de almidón de reserva. Unos de forma circular de tamaño promedio de 19,9 $\mu \mathrm{m}$, con hendidura radial, delicada, de hilio central, distintivo (Fig. 3A), que a la luz polarizada muestra una cruz céntrica, simétrica y distintiva. Otros ovalados, de dos tama- ños, los más grandes de tamaño promedio de $33,8 \mu \mathrm{m}$ de largo x 21,5 $\mu \mathrm{m}$ de ancho y los más pequeños con tamaño promedio de 22,5 $\mu \mathrm{m}$ de largo x $15 \mu \mathrm{m}$ de ancho, de hilio indistintivo, presentando además una fisura longitudinal ramificada. Observado con luz polarizada se presenta una cruz excéntrica, asimétrica y distintiva (Fig. 3B y 3C). 

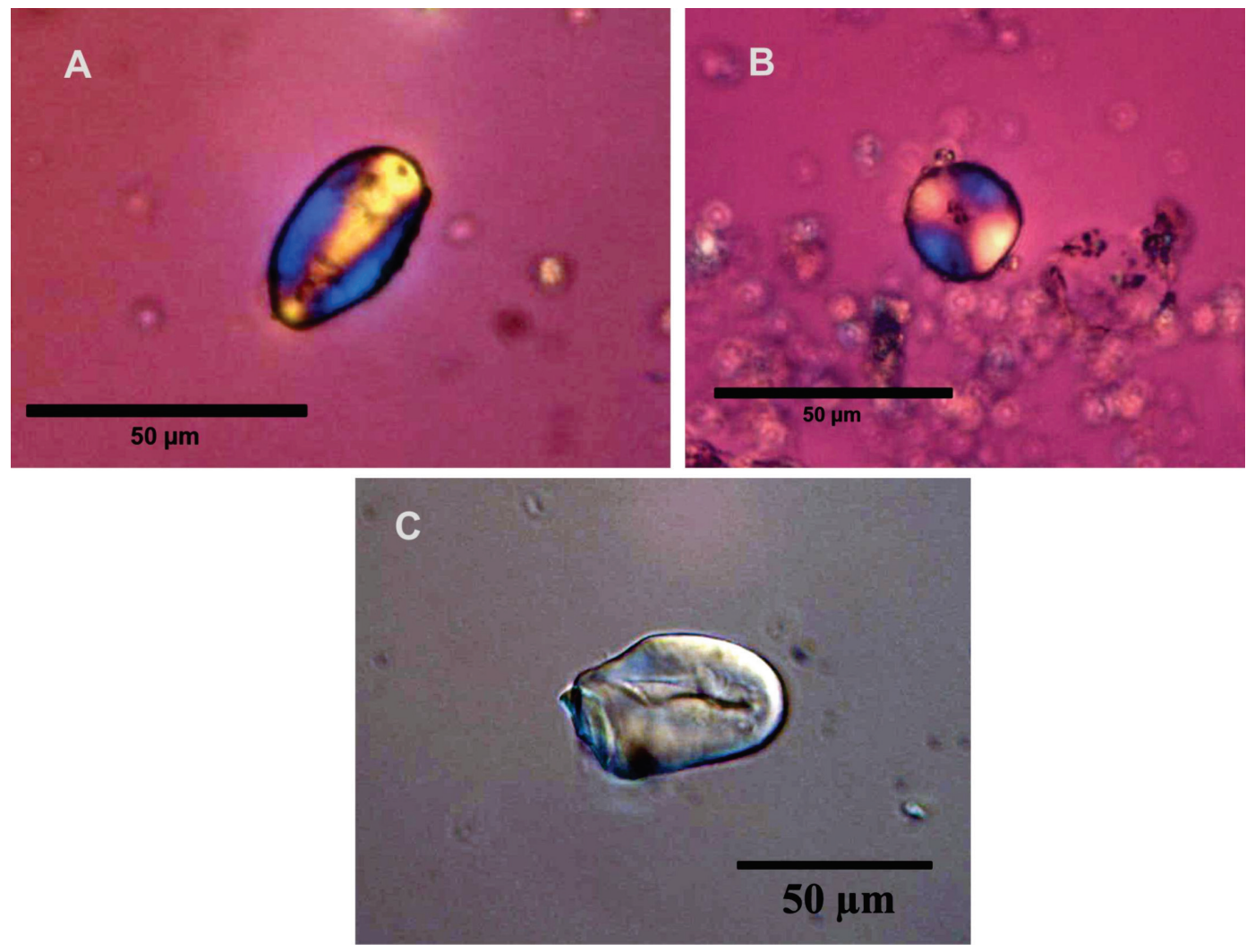

Fig. 3. Nicotiana paa. A] Grano de almidón circular. B] Grano de almidón ovalado. C) Grano de almidón ovalado (DIC). Scarpa 798 (BA).

\section{DISCUSIÓN Y CONCLUSIONES}

Como se mencionara anteriormente, el «coro» constituyó una incógnita respecto de su identidad botánica hasta que el trabajo pionero de Martínez Crovetto registró su empleo en un grupo reducido de moqoit del Chaco argentino.

El estudio anatómico realizado en el rizoma de «coro» evidenció, a través del corte transversal, una estructura de tallo secundario subterráneo, caracterizado por la presencia de súber. Un parénquima de reserva, con una abundante cantidad de almidón indica que una de las funciones principales de este órgano es la de reserva. La disposición del tejido conductor en un sistema vascular continuo con presencia de cambium entre floema y xilema denota su carácter de Dicotiledónea.
En el disociado de la corteza del rizoma se observaron idioblastos cristalíferos (que contienen arenillas cristalinas) comunes en las Solanáceas (Metcalfe y Chalk, 1979). La presencia de súber denota su carácter secundario y se determinaron además la presencia de esclereidas, rectangulares y fibroesclereidas. Por otro lado, en el polvo de la corteza se observaron dos tipos de granos de almidón: redondeados, con hendidura e hilio central y ovalados, de hilio excéntrico. En lo que respecta al leño, se observaron abundantes fibras, unidades de vaso de tipo reticulado y células parenquimáticas, elementos característicos de este tejido.

La identificación de las estructuras microscópicas presentes, y en especial los granos almidón, resultan de mucha utilidad como herramienta diagnóstica a partir del 
análisis de sus restos en otros objetos de la cultura material moqoit.

Este trabajo constituye el primer análisis morfo-anatómico de este órgano subterráneo, el cual es empleado como masticatorio y/o fumatorio entre distintos pueblos originarios chaqueños, principalmente los moqoít y qom, como así también por otros pueblos originarios y es un punto de partida para futuros estudios de etnobotánica y paleoetnobotánica que incluyan a distintos grupos humanos que puedan haber hecho empleo de este vegetal.

\section{AGRADECIMIENTOS}

A la Universidad de Buenos Aires (Proyecto UBACYT 2014-2017 No 20020130100641BA) y al Consejo Nacional de Investigaciones Científicas y Técnicas de Argentina (CONICET).

\section{BIBLIOGRAFÍA}

Boodle, L. A. (1916). A method of macerating fibres. Kew Bulletin of miscellaneous information 4: 108-110.

Cárdenas, M. (1968). Masticatorios y fumatorios. XXXVII congreso internacional de Americanistas. Actas y Memorias, Vol. II. Buenos Aires.

D’Ambrogio de Argüeso, A. (1986). Manual de técnicas en histología vegetal. Buenos Aires, Argentina: Hemisferio sur.

Deckmann Fleck, E. C. (2015). As Artes de curar em um manuscrito jesuítico inédito do setecentos. O Paraguay Natural Ilustrado do padre José Sánchez Labrador (17711776). São Leopoldo, Brasil: Oikos.

Dobrizhoffer, M. [(1784] 1967]. Historia de los abipones. Vol. 1. Resistencia, Argentina: Universidad Nacional del Nordeste.

Farmacopea Argentina séptima edición, vol. III. Recuperado de http://www.anmat. gov.ar/webanmat/fna/pfds/Libro_Tercero.pdf.

ICSN (2011). The International Code for Starch Nomenclature. Recuperado de http://fossilfarm.org/ICSN/Code.html.

Jolis, J. [(1789) 1972]. Ensayo sobre la historia natural del Gran Chaco. Resistencia, Argentina: Universidad Nacional del Nordeste
Lozano, P. [(1754-1755] 1875]. Historia de la conquista del Paraguay, Rio de la Planta y Tucumán. Vol 5. Buenos Aires, Argentina: Imprenta popular Bs. As.

Martinez Crovetto, R. (1978). Una nueva especie de Nicotiana de la flora argentina. Bonplandia 5 (2): 7-10.

Martinez Crovetto, R. (1980). Identificación botánica del "coro", antiguo fumatorio utilizado por los indios del Chaco (Republica Argentina). En: Editores Mexicano Unidos (Eds.). La antropología Americanista en la Actualidad. Homenaje a Raphael Girard. Tomo 2 (pp. 455-463). México.

Metcalfe, C. R. y Chalk, L. (1979). Anatomy of the Dicotyledons, systematic anatomy of the leaves and stems. Vol. II. Second Edition. Oxford, England: Clarendon Press.

Métraux, A. (1946). Ethnography of the Chaco. En: Steward. Smithsonian Institution (Eds). Handbook of South American Indians 1 (pp. 197-370) Washington, USA.

Montenegro, P. (1945), Materia Médica Misionera. Buenos Aires, Argentina: Imprenta de la Biblioteca Nacional.

Pérez Gollán, J. A. y Gordillo, I. (1993). Alucinógenos y sociedades indígenas del noroeste argentino. Anales de Antropología 30: 299-350.

Ruiz Moreno, A. (1948). La medicina en "el Paraguay Natural" (1771-1776) del P. José Sánchez Labrador s. j. Tucumán, Argentina: Universidad Nacional de Tucumán.

Scarpa, G. F. y Rosso, C. N. (2011). Etnobotánica del "coro" (Nicotiana paa, Solanaceae): Un tabaco silvestre poco conocido del extremo sur de Sudamérica. Bonplandia 20 (2): 391-404.

Schulz, A. G. (1976). Nombres comunes de las plantas. Colonia Benítez, Argentina: Talleres Gráficos Moro.

Serrano, A. (1934). El uso del tabaco y vegetales narcotizantes entre los indígenas de américa. Revista Geográfica Americana 2 (15): 415-430.

Zardini, E. (1976-1977). The identification of an Argentinian Narcotic. Botanical Museum Leaflets. 25 (3). Cambridge, USA: Harvard University.

Zarlavsky, G. [2014]. Histología Vegetal. Técnicas simples y complejas. Buenos Aires, Argentina: Sociedad Argentina de Botánica. 